\title{
MEGAMASERS AND THEIR HOST GALAXIES
}

\author{
Willem A. Baan \\ ASTRON - Westerbork Observatory, POBox 2, 7990 AA Dwingeloo, \\ The Netherlands
}

\begin{abstract}
The variety of molecular megamasers trace different classes of galaxies and provide information on their various nuclear components. $\mathrm{H}_{2} \mathrm{O}$ megamaser regions may trace the inner parsec-scale regions with keplerian disks of the LINER and Seyfert 2 nuclei, which are relatively naked and not dominated by dust or starforming regions. The $\mathrm{OH}$ and $\mathrm{H}_{2} \mathrm{CO}$ megamaser emissions exhibit the physics and dynamics of the inner few hundred parsecs of ultra-luminous FIR galaxies, which are dusty, compact, and whose perturbed nuclei are powered by compact starbursts.
\end{abstract}

\section{Introduction}

The megamaser phenomenon studied extensively in recent years has made it clear that these molecular emissions provide valuable tools for the study of the nuclear regions of active galaxies. Of the four megamaser types the $\mathrm{OH}$ and $\mathrm{H}_{2} \mathrm{O}$ ones have been studied most widely. The goal of this review is to highlight some high-resolution studies of megamasers and their systematic trends.

\section{2. $\mathrm{OH}$ and $\mathrm{H}_{2} \mathrm{CO}$ Megamasers}

Only the most outstanding sources among the about 90 known $\mathrm{OH}$ megamasers ( $\mathrm{OH} \mathrm{MM})$ have been studied at sub-arcsecond resolution. Among the sources studied in detail are: Arp 220 (Diamond et al. 1989; Lonsdale et al. 1998; Smith et al. 1998), III Zw 35 (Montgomery \& Cohen 1992; Trotter et al. 1997; Diamond et al. 1999), IR 17208-0014 (Diamond et al. 1999), Mrk 231 (Lonsdale et al., 1998; Klöckner et al. 2001), Mrk 273 (Yates et al. 2000; Klöckner et al. 2001), and IC 694/NGC 3690 (Polatidis et al. 2000; Klöckner et al. 2001).

The high-resolution studies recover a fraction of the total $\mathrm{OH}$ flux in the high brightness components of the megamasers ranging from about $65 \%$ for Arp 220 to only $20 \%$ for IR $17208-0014$. Besides the compact VLBI components, there must be a significantly extended component that will be resolved at 50 to $500 \mathrm{~km}$ baselines using large telescopes.

The emission regions found in $\mathrm{OH} \mathrm{MM}$ can be divided into two categories. First, extended, weak emission that results from unsaturated masering and amplification of background continuum. The pumping agent for these regions is likely the FIR radiation field. The radio continuum that is being amplified is often resolved with VLBI baselines and a good amplification scenario is not yet available. Second, compact high brightness components resulting from saturated 
masering with gains between 100 and 800 . It still appears that even with those high-gain components the background continuum may play a role. No continuum superposition has been seen with VLBI studies. The pumping agent for this type of high-gain emission could be shocks but the FIR radiation field could play a significant role too.

Because of the general weakness of the $\mathrm{H}_{2} \mathrm{CO}$ emission lines at $4.8 \mathrm{Ghz}$, there has been only one study of Arp 220 (Baan \& Haschick 1995).

\section{1. $\mathrm{OH}$ and $\mathrm{H}_{2} \mathrm{CO}$ Studies of Arp 220}

Arp 220 (IC 4553) has been the best studied example of a powerful MM host galaxy, which is also prominent in other molecular line and FIR/NIR emissions. The earliest sub-arcsec high-resolution observations with MERLIN showed that the line emission was indeed confined to the two nuclei (Diamond et al. 1989). Recent high resolution global VLBI has revealed clusters of about a dozen powerful SNRs at each of the nuclei, that account for about $3 \%$ of the continuum flux of the nuclei (Smith et al. 1998). Pairs of extended $\mathrm{OH}$ emission regions were found straddling the SNR clusters at each of the nuclei, and containing embedded high brightness components (Lonsdale et al. 1998). Recent $6 \mathrm{~cm}$ observations with MERLIN show two elongated continuum structures in Arp 220, with central $6 \mathrm{~cm}$ contours agreeing in shape and size with the two SNR clusters (Baan, Klöckner, \& Cohen 2001).

The $\mathrm{OH}$ emission regions detected at the SE nucleus are at the same velocity as the emission at the NW nucleus (see also Baan, Klöckner, \& Cohen 2001). While this clearly hints at molecular gas associated with the NW being present in the nuclear region of the $\mathrm{SE}$, the data does confirm that two emission line pairs at 1667 and $1665 \mathrm{MHz}$ are emitted by the two nuclei of Arp 220 (Baan \& Haschick 1984). The emission at the velocity of the NW nucleus is much more prominent than the emission at the velocity of the SE nucleus. The $1667 \mathrm{MHz}$ emission of the SE nucleus could be found with broad band observations, that include the much weaker $1665 \mathrm{MHz}$ line of both the NW and SE nucleus.

The studies of the $4 \mathrm{mJy}$ line of $\mathrm{H}_{2} \mathrm{CO}$ in Arp 220 at sub-arcsecond resolution are complementary to the $\mathrm{OH}$ and other molecular studies. The VLA-A observations recover all of the flux in the $\mathrm{H}_{2} \mathrm{CO}$ line and reveal that the emission is confined to the two nuclei and the area in between. The velocity of most of the emission is again similar to that of the NW nucleus and it peaks at the $\mathrm{NW}$ nucleus (Baan \& Haschick 1995). The $\mathrm{H}_{2} \mathrm{CO}$ emission structure is almost identical to that of the NIR emission across the whole nuclear region, as seen in subsequent NICMOS observations (Scoville et al. 1998). The $\mathrm{H}_{2} \mathrm{CO}$ and CO emission data have made it possible to model the dynamics of the Arp 220 nuclear system (Baan \& Haschick 1995; Scoville et al. 1997, Sakamoto et al. 1999).

The emission in the $4.8 \mathrm{GHz}$ formaldehyde line appears to be diffuse, unsaturated, and amplifying the background continuum. The close similarity with the NIR emission raises the possibility that the infrared radiation field serves as a pump for the $\mathrm{H}_{2} \mathrm{CO}$ maser action. Earlier suggestions for the pump were based on the radio continuum. 

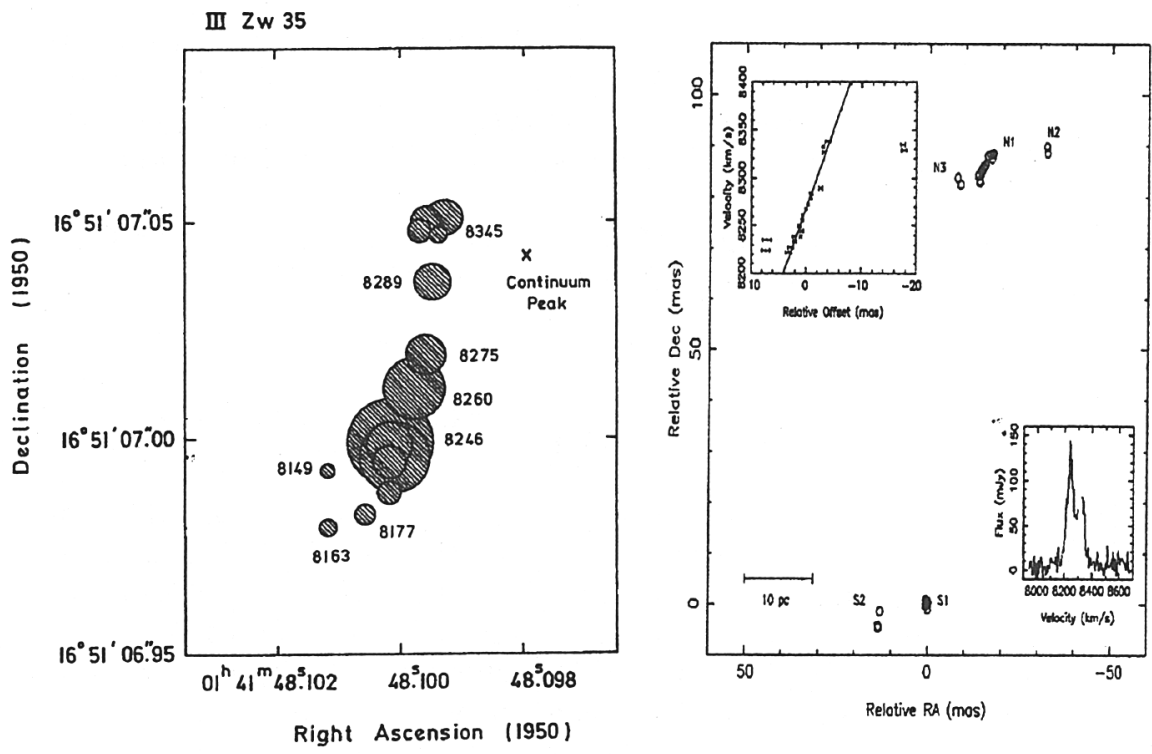

Figure 1. The spatial distribution of $\mathrm{OH}$ maser components in III Zw 35. a) The MERLIN data (Montgomery \& Cohen 1989), b) Global VLBI data from Diamond et al. (1999).

Recent observations with MERLIN have provided a continuum map for Arp 220 but have failed to detect the $\mathrm{H}_{2} \mathrm{CO}$ line emission (Baan, Klöckner, \& Cohen 2001). The next best formaldehyde candidate is NGC 6240 .

\subsection{OH Emission in III Zw 35}

The masering regions III Zw 35 show a total extent of about 50 pc with some fifteen components surrounding the nucleus of the galaxy from south to east (Montgomery \& Cohen 1992: Figure 1). Higher resolution studies reveal two clusters of bright components of about $10 \mathrm{pc}$ in extent, which constitute together about $50 \%$ of the $1667 \mathrm{MHz}$ line flux (Trotter et al. 1997; Diamond et al. 1999: Figure 1). The northern components account for the "high-velocity horn" of the single dish profile and the southern component for the stronger "low-velocity horn". The estimated gain of the brightest maser components is approximately 500 and could be higher if better upper limits for the continuum are used.

The location of the emission regions and the velocity structure of the MERLIN data would suggest that the molecular emission regions are part of a $100 \mathrm{pc}$ inclined disk centered on the radio nucleus (Montgomery \& Cohen 1992). However, each of these two compact regions shows velocity gradients, which cannot be easily interpreted in terms of this same disk structure and suggest that other dynamic forces are at work in the emission regions. 


\section{3. $\mathrm{H}_{2} \mathrm{O}$ Megamasers}

The known $\mathrm{H}_{2} \mathrm{O}$ MMs are still relatively nearby and most of the total flux occurs in high brightness components, but the number of VLBI sources is relatively larger than that of $\mathrm{OH} \mathrm{MMs}$. Sources that have been studied in detail are: NGC 1052 (Claussen et al. 1998), NGC 1068 (Greenhill et al. 1996; Gallimore et al. 1996), NGC 3079 (Baan \& Irwin 1995; Trotter, et al. 1998; Sawada-Satoh et al. 2000), NGC 4258 (Myoshi et al. 1995; Greenhill et al. 1995), and NGC 5793 (Hagiwara et al. 1997).

It is not clear whether just any Seyfert 2 galaxy emits maser emission with a certain anisotropy or whether maser action requires some special physical circumstances subject to evolutionary processes. At present the success rate for finding megamasers in directed searches has been relatively low (some 6\%; see Braatz, Wilson \& Henkel 1997) with some 350 galaxies searched but only 21 megamasers found.

The great resolving power obtained with VLBI at $22 \mathrm{GHz}$ provides a detailed view of $\mathrm{H}_{2} \mathrm{O}$ megamaser nuclei corresponding to a spatial resolution of less than $0.1 \mathrm{pc}$ for the nearby sources. There appear to be two types of $\mathrm{H}_{2} \mathrm{O} \mathrm{MMs}$, which are distinct in their pumping mechanisms and emission structures. Type 1 sources are Syferts or LINERs with edge-on nuclear disks that may exhibit the keplerian motion in the disk and the centripetal acceleration of the emission components (see Wilson 1998 for inventory). The dynamics of some central pcscale molecular disks have been revealed indicating central masses ranging from $1 \times 10^{6} \mathrm{M}_{\odot}$ for NGC 4945 and NGC 3079 to respectively 17 and $36 \times 10^{6} \mathrm{M}_{\odot}$ for NGC 1068 and NGC 4258. Type 2 sources have broad $\mathrm{H}_{2} \mathrm{O}$ emission profiles originating not in the nucleus itself but rather along the nuclear jets.

The compact high brightness components are thought to be saturated maser pockets pumped with nuclear X-rays for the type 1 sources, while shock-pumping has been suggested for the type 2 sources. It should be noted that in some sources the strongest $\mathrm{H}_{2} \mathrm{O}$ maser components are superposed on the radio continuum. The presence of background continuum could render maser components with lower gain observable. If amplification plays a partial role in the masering process, as indicated in the above sections, the $\mathrm{H}_{2} \mathrm{O}$ maser luminosity would vary as $\left(\mathrm{L}_{\text {radio }} \mathrm{xL}_{x-\text { ray }}\right)$. Within the uncertainty of the $\mathrm{X}$-ray luminosities, some correlation has indeed been found for this relation (Baan 1997).

In the next section, three sources will be reviewed that provide an overview of the physics of megamaser emission regions. The prototype type 1 source NGC 4258 has been discussed extensively elsewhere.

\subsection{NGC 3079 - type 1}

The $\mathrm{H}_{2} \mathrm{O}$ maser emission in $\mathrm{NGC} 3079$ is part of a complex but consistent picture of a compact masering disk tilted by $42^{\circ}$ relative to a radio triple jet structure and aligns roughly with the disk of the galaxy (Baan \& Irwin 1995; Sawada-Satoh et al. 2000; Trotter et al. 1998). Strong nuclear OH and HI absorption occurs against the triple jet source. Detailed mapping of the $\mathrm{H}_{2} \mathrm{O}$ maser components indicate clear rotation in the $2 \mathrm{pc}$ disk and shows that the maser components are not superposed on strong compact radio sources (Figure 


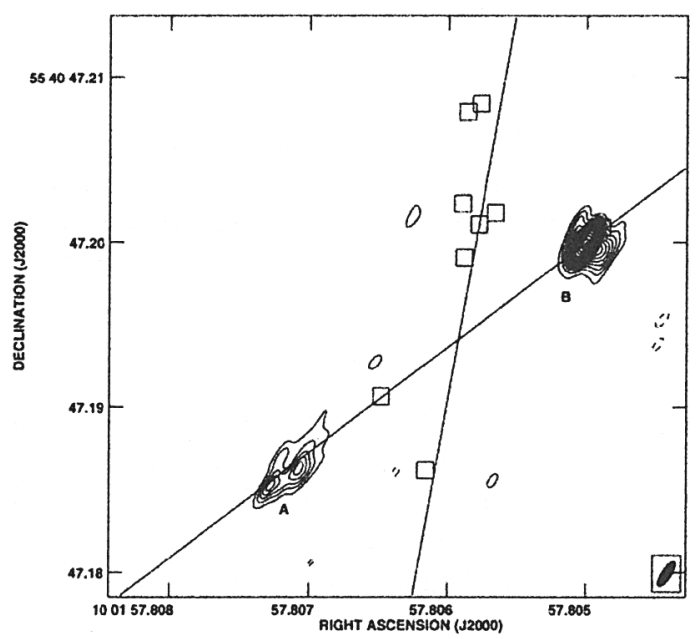

Figure 2. The positions of the $\mathrm{H}_{2} \mathrm{O}$ maser components relative to the continuum components in NGC 3079 (Trotter et al. 1998). The axis of the galactic disk aligns roughly with the maser disk.

2). Since NGC 3079 displays a nuclear super-bubble and blow-out, the maser pumping could be related to shocks in a nuclear wind as well.

\subsection{NGC 1068 - type 1 and type 2}

The maser components at the nucleus of NGC 1068 trace a slightly curved structure, which constitutes a full quarter of an edge-on thin keplerian disk with a clear turnover and with an inner and outer radius of about $0.65 \mathrm{pc}$ and $1.1 \mathrm{pc}$ (Gallimore et al. 1996; Greenhill et al. 1996). The disk is inclined some $-45^{\circ}$ relative to the nuclear jet. The $\mathrm{H}_{2} \mathrm{O}$ emission in NGC 1068 extends over more than $750 \mathrm{~km} \mathrm{~s}^{-1}$ with individual components showing strong variability. All these type $1 \mathrm{H}_{2} \mathrm{O}$ maser components appear superposed on the radio continuum of the nuclear region.

In addition, NGC 1068 shows some type $2 \mathrm{H}_{2} \mathrm{O}$ masers superposed on a knot in the nuclear jet at a distance of $50 \mathrm{pc}$ from the nucleus (Gallimore et al. 1996). There are also $\mathrm{OH}$ masers and $\mathrm{HI}$ absorption at the nucleus, which may serve as probes for the torus structure.

\subsection{NGC 1052 - type 2}

NGC 1052 and its look-alike TXFS 2226-184 are elliptical galaxies with double nuclear jets and broad emission spectra. The maser components in NGC 1052 lie along the jet rather than perpendicular to it and show a clear velocity gradient. The molecular gas appears to be part of a large-scale foreground structure rather than part of a torus (Claussen et al. 1998). Two masering regions can be decomposed in some 40 high brightness components. The type 2 maser emission in NGC 1052 appears again associated with shock heating and with the background continuum. However, the nature of the regions is not clear because the 

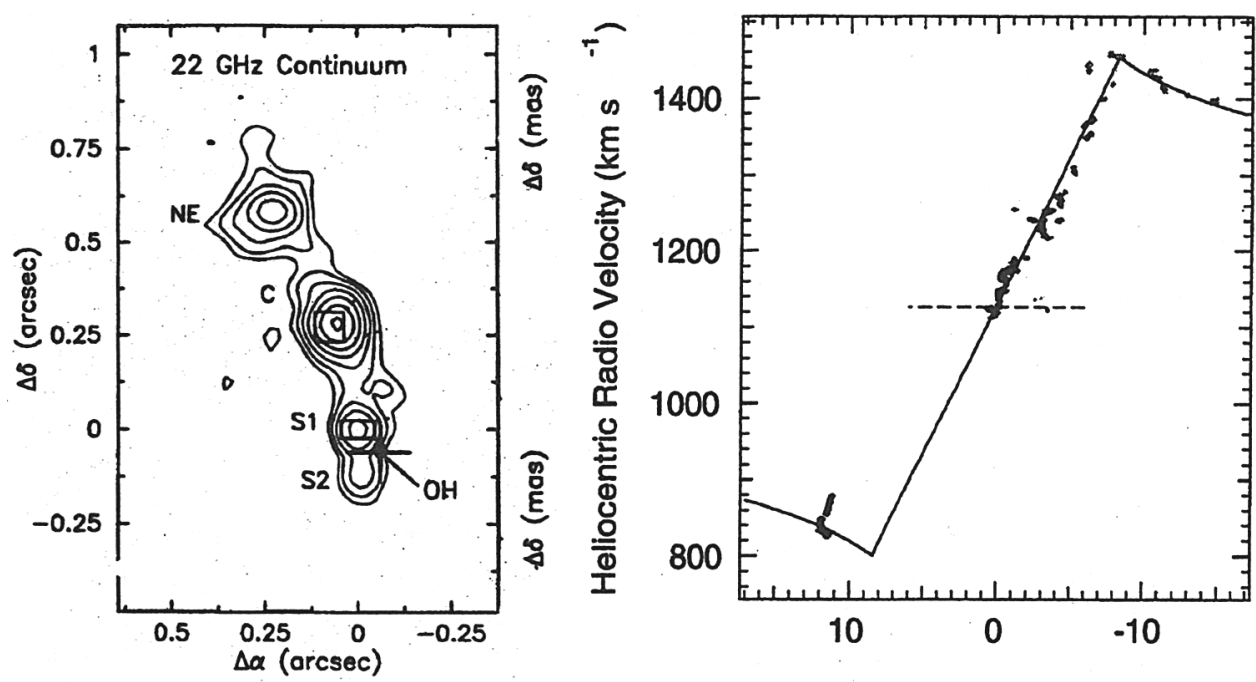

Impact Parameter (mas)

Figure 3. The continuum structure of NGC 1068 from Gallimore et al. 1996. Type $1 \mathrm{H}_{2} \mathrm{O}$ masers occur at the nuclear position $\mathrm{S} 1$, while type 2 components occur along the jet at position of the knot $\mathrm{C}$ (Gallimore et al. 1996). OH masers occur southwest of the nucleus . The keplerian rotation curve of the disk components has been given in the right frame (Greenhill \& Gwinn 1996).

maser regions could be associated with (shock-) interaction regions but could also be gas clouds entrained into the jet flow.

\section{Nature of the Host Galaxies}

The known $\mathrm{OH}$ and $\mathrm{H}_{2} \mathrm{O}$ MM populations have distinct characteristics when using the FIR luminosities as a comparison basis (Baan 1997). The fraction of $\mathrm{OH}$ megamasers among the FIR population increases with FIR luminosity and varies from $10 \%$ to $50 \%$ for the ultra-luminous galaxies. This increase reflects the combined effects of increasing pumping efficiency and increasing opening-angle for the masering process with the FIR luminosity.

Contrary to the $\mathrm{OH}$ host galaxies, the fraction of $\mathrm{H}_{2} \mathrm{O}$ MMs among the AGN/Seyfert 2 population decreases with increasing FIR luminosity from $8 \%$ to less than $1 \%$ at $\mathrm{L}_{F I R} \geq 10^{10} \mathrm{~L}_{\odot}$. An anti-correlation of $\mathrm{H}_{2} \mathrm{O}$ maser activity and FIR luminosity is consistent with an other-than-FIR pumping agent for $\mathrm{H}_{2} \mathrm{O}$ and the requirement of non-dusty (naked) AGN's for $\mathrm{H}_{2} \mathrm{O}$ maser activity. The anti-correlation confirms again that the molecular structure associated with the $\mathrm{OH}$ and $\mathrm{H}_{2} \mathrm{O}$ maser activity is different.

Host galaxies of $\mathrm{OH}$ and $\mathrm{H}_{2} \mathrm{CO}$ megamasers have been intensely studied as part of the ULIRG population. There is a discrepancy between the optical classification of these sources showing predominantly LINER and Seyfert charac- 
teristics and their radio classification showing starburst characteristics (Condon 1992; Baan et al. 1998, 2001). A mixing sequence can be constructed for $\mathrm{OH}$ host galaxies running from pure nuclear starburst to pure active galactic nucleus in order to explain the radio and optical characteristics of $\mathrm{OH}$ and $\mathrm{H}_{2} \mathrm{CO}$ host galaxies (Baan et al. 2001). This mixing sequence is similar to that for the NIR properties of ULIRGs and prominent OH host galaxies (Genzel et al. 1998). The radio and NIR classification appears most representative of the nuclear activity.

The $\mathrm{H}_{2} \mathrm{O}$ MM host galaxies are medium radio-loud with low FIR-to-radio luminosity ratios. (Baan 1997). Only few Seyfert 1 and 2 sources among the $\mathrm{OH}$ galaxies are radio-loud, while other $\mathrm{OH}$ hosts are radio-weak.

\section{Conclusions}

High-resolution interferometric observations of masering nuclei provide a independent diagnostic tool to study the nature and dynamics of active nuclei and starburst nuclei. In $\mathrm{OH}$ sources both compact and extended components are found, while a large fraction of the line emission has been overresolved. The $\mathrm{OH}$ maser activity serves as an important signpost of ULIRG and SBN activity for a large population of sources that follows the galaxy merger rate. For $\mathrm{OH}$ and $\mathrm{H}_{2} \mathrm{CO}$ there is a valuable connection with the NIR/FIR and other molecular emissions. In $\mathrm{H}_{2} \mathrm{CO}$ maser regions there is no evidence yet of very compact components. A comparison of results has been given in Table 1.

Table 1. High Resolution View of Megamaser Galaxies

\begin{tabular}{|l|l|l|l|}
\hline \hline Parameter & $\mathrm{OH}$ & \multicolumn{1}{l|}{$\mathrm{H}_{2} \mathrm{CO}$} & $\mathrm{H}_{2} \mathrm{O}$ \\
\cline { 1 - 2 } Compact mas components & yes & no & yes \\
VLBI 10 mas structure & yes & yes & no \\
VLBI 100 mas structure & yes & yes & no \\
Pumping mechanism & FIR/shocks & NIR? & X-ray/shocks \\
Amplification & yes/no & yes & yes/no \\
Spatial structure & dusty torus & dusty torus & compact disk \\
& & & shock regions \\
Dynamical View & $10-100 \mathrm{pc}$ & $10-100 \mathrm{pc}$ & $0.1-3 \mathrm{pc}$ \\
\hline
\end{tabular}

The $\mathrm{H}_{2} \mathrm{O}$ MMs display mostly unresolved high-brightness point sources, which recover a large fraction of the single dish flux. Type 1 sources reveal the dynamics of nuclear disks (few pc) with a range of $\mathrm{BH}$ masses, They are likely pumped by X-rays. Type 2 maser regions may reveal jet interaction regions and are pumped by shocks.

The molecular megamasers trace different galaxy populations with redshift. Given a sufficient sensitivity for the global VLBI systems, the disks of $\mathrm{H}_{2} \mathrm{O}$ sources may be resolved out to redshifts of 0.06 . On the other hand, the larger scale $\mathrm{OH}$ regions may be resolved out to redshifts of 0.9 and well into the redshift range of interest for galaxy evolution.

Several outstanding questions remain regarding the masering activity in galactic nuclei. Further study is needed for the pumping mechanisms of the 
molecules as well as their connection to the amplification of background continuum, since it plays a role in a significant fraction of the emission regions for $\mathrm{OH}$ and for both types of $\mathrm{H}_{2} \mathrm{O}$ masers. The discrepancy between the optical and radio characteristics of $\mathrm{OH}$ and $\mathrm{H}_{2} \mathrm{CO}$ sources and the (modified) nuclear environment of ULIRGs requires further study. Finally, there is a need to employ wide-field mapping techniques to recover the extended emission structures.

\section{References}

Baan, W.A. 1997, in High Sensitivity Radio Astronomy, (Cambridge), 73

Baan, W.A. \& Haschick, A.D. 1984, ApJ, 279, 541

Baan, W.A. \& Haschick, A.D. 1995, ApJ, 454, 745

Baan, W.A. \& Irwin, J.A.. 1995, ApJ, 446, 602

Baan, W.A., Klöckner. H.-R., et al. 2001, ApJ, submitted

Baan, W.A., Klöckner. H.-R., Cohen, R.J. 2001, in preparation

Baan, W.A., Salzer, J.J., \& LeWinter, R.D. 1998, ApJ, 509, 633

Braatz, J.A., Wilson, A.S., \& Henkel, C. 1997, ApJS, 110, 321

Claussen, M.J., Diamond, P.J., Braatz, J.A., et al. 1998, ApJ, 500, 129

Condon, J.J. 1993, in Protostars to Protogalaxies, ASP Conf. Ser, 43, 87

Diamond, P., Lonsdale, C., Lonsdale, C., \& Smith, H. 1999, ApJ, 511, 178

Diamond, P.J., Norris, R.P., Baan, W.A., \& Booth, R.S. 1989, ApJ, 340, L49

Gallimore, J., Baum, S., O'Dea, C. et al. 1996, ApJ, 462, 740

Genzel. R. et al. 1998, ApJ, 498, 579

Greenhill, L.J., \& Gwinn, C.R. 1997, Ap\&SS, 248261

Greenhill, L., Gwinn, C., Antonucci, R., \& Barvainis, R. 1996, ApJ, 472, L21

Greenhill, L.J., Jiang, R.D., Moran, J.M., et al. 1995, ApJ, 440, 619

Hagiwara, Y., Kohno, K., Kawabe, R, \& Nakai, N. 1997, PASJ, 49, 171

Klöckner, H.-R., et al. 2001, in preparation

Lonsdale, C.J., et al. 1998, unpublished

Lonsdale, C., Diamond, P., Smith, H., \& Lonsdale, C., 1998, ApJ, 493, L13

Montgomery, A.S. \& Cohen, R.J. 1992, MNRAS, 254, 23P

Myoshi, M. et al. 1995, Nature, 373, 127

Polatidis, A. et al. 2000, these proceedings

Sakamoto, K., Scoville, N.Z., et al. 1999, ApJ, 514,68

Sawada-Satoh, S., Inoue, M., Shibata, K.M., et al. 2000, PASJ, 52, 421

Scoville, N.Z., Yun, M.S., \& Bryant, P.M., 1997, ApJ, 484, 702

Scoville, N.Z., et al. 1998, ApJ, 492, L107

Smith, H., Lonsdale, C., Lonsdale, C., \& Diamond, P. 1998, ApJ, 493, L17

Trotter, A.S., Moran, J.M., Greenhill, L.J., et al. 1997 ApJ, 485, L79

Trotter, A.S., Greenhill, L.J., Moran, J.M., et al. 1998, ApJ, 495, 740

Wilson, A.S. 1998, in Some Like it Hot, AIP Conf. Proc., 431, 235

Yates, J.A. Et al. 2000, MNRAS, 317, 28 